Supplement of Hydrol. Earth Syst. Sci., 22, 2839-2865, 2018

https://doi.org/10.5194/hess-22-2839-2018-supplement

(C) Author(s) 2018. This work is distributed under

the Creative Commons Attribution 4.0 License.

(c) (1)

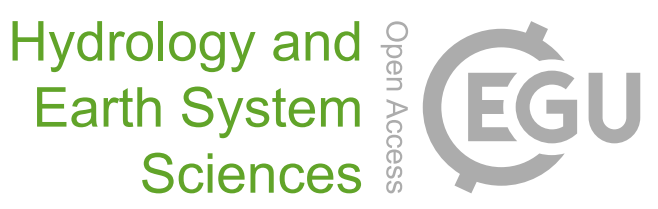

Supplement of

\title{
Basin-scale impacts of hydropower development on the Mompós Depression wetlands, Colombia
}

Héctor Angarita et al.

Correspondence to: Héctor Angarita (hangarita@javeriana.edu.co)

The copyright of individual parts of the supplement might differ from the CC BY 4.0 License. 


\section{SI-1. SUPPLEMENTARY INFORMATION}

\section{ReservoirSimulator model description}

The ReservoirSimulator allows simulation of the water balance of a group of reservoirs, based on individual or system-level dispatch rules for three main categories of use: downstream instream requirements, hydropower, and other supply (supply that 5 bypasses turbines).

For a given reservoir, the model takes into account physical and technical constraints, such as the volume-elevation curve, tail-water elevation, operational levels (inactive, buffer, technical, and safety), turbine type, capacity, and efficiency. It allows modeling of the topology of reservoirs and tributary sub-basins.

ReservoirSimulator performs a lumped, discrete-time water balance of the components shown in Figure SI-1 with the model

10 parameters and inputs shown in Table SI-1.

For each reservoir, calculations are done according to the sequence in Table SI-2:

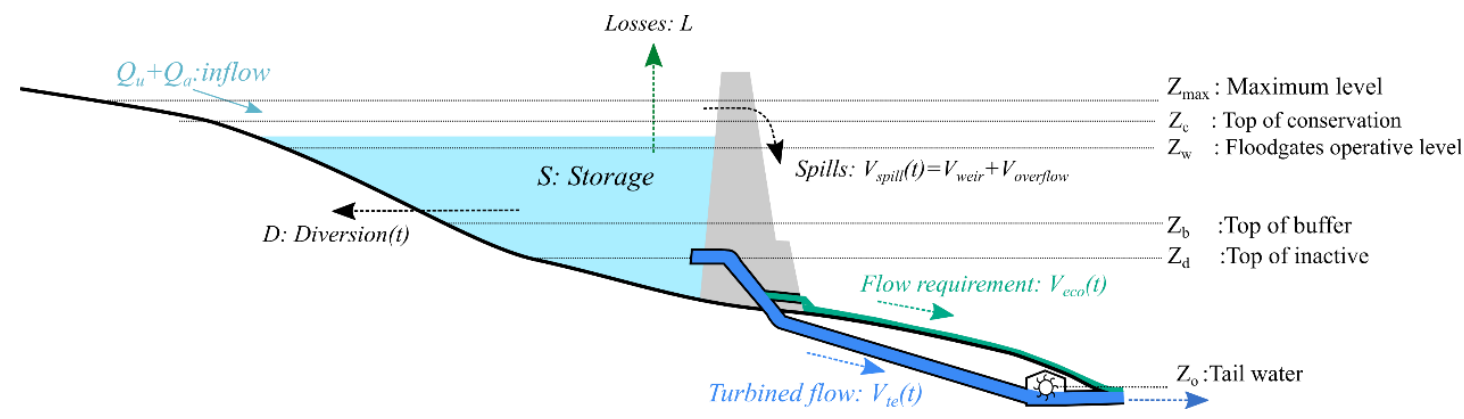

Figure SI-1. Schematic of reservoir inflow, outflow and storage components of the reservoir simulator model. 
Table SI-1. Description of reservoir data requirements of the model

\begin{tabular}{|c|c|c|}
\hline Symbol & Reservoir physical and technical data & Units \\
\hline $\mathrm{Q}_{\mathrm{a}}(\mathrm{t})$ & $\begin{array}{l}\text { Specific runoff: Runoff from upstream sub-watershed during step } t \text { (excludes outflows from } \\
\text { upstream infrastructure) }\end{array}$ & $\mathrm{m}^{3} / \mathrm{s}$ \\
\hline$\Delta \mathrm{t}$ & Time step & $\mathrm{s}$ \\
\hline $\mathrm{V}_{\mathrm{c}}$ & Reservoir storage capacity at top of conservation level $\left(\mathrm{Z}_{\mathrm{c}}\right)$ & $\mathrm{m}^{3}$ \\
\hline $\mathrm{V}_{\text {buffer }}$ & Reservoir storage capacity at buffer level $\left(\mathrm{Z}_{\mathrm{b}}\right)$ & $\mathrm{m}^{3}$ \\
\hline $\mathrm{V}_{\min }$ & Reservoir storage capacity at top of inactive level $\left(\mathrm{Z}_{\mathrm{d}}\right)$ & $\mathrm{m}^{3}$ \\
\hline $\mathrm{V}_{w 0}$ & Reservoir storage capacity at floodgates operative level $\left(\mathrm{Z}_{\mathrm{w}}\right)$ & \\
\hline $\mathrm{H}(\mathrm{V})$ & Volume-elevation curve of reservoir & masl \\
\hline$A(V)$ & Area-elevation curve of reservoir & masl \\
\hline $\mathrm{B}_{c}$ & $\begin{array}{l}\text { Buffer coefficient: fraction of buffer storage that can be allocated in a given time step } \\
\text { Note: Can be a user defined function of local (i.e. reservoir storage, etc.) or system-level state } \\
\text { variables or context variables (i.e. downstream storage, etc.). }\end{array}$ & $\%$ \\
\hline $\mathrm{B}_{w}$ & $\begin{array}{l}\text { Floodgates allocation factor: determines the fraction of storage above the floodgates } \\
\text { operational level delivered downstream in a given time step. } \\
\text { Note: Can be a user defined function of local (i.e. reservoir storage, etc) or system-level state } \\
\text { variables or context variables (i.e. downstream storage, etc.). }\end{array}$ & \\
\hline $\mathrm{ET}(\mathrm{t})$ & Evaporation rate in a time step $t$ & $\mathrm{~mm}$ \\
\hline $\mathrm{Z}_{0}$ & Tail water elevation (water level at the point where turbine flow is discharged) & masl \\
\hline $\mathrm{D}(\mathrm{t})$ & $\begin{array}{l}\text { Water demand diverted from the reservoir, bypassing the turbines, with higher priority than } \\
\text { hydropower }\end{array}$ & $\mathrm{m}^{3}$ \\
\hline $\mathrm{V}_{\mathrm{eco}, 1}(t)$ & $\begin{array}{l}\text { Average instream flow requirement at step } t \text {, for the reach between reservoir and turbines } \\
\text { discharge }\end{array}$ & $\mathrm{m}^{3}$ \\
\hline $\mathrm{P}$ & Installed generation capacity & Mw \\
\hline f & Friction loss factor in hydropower load pipes. & $\mathrm{m}$ \\
\hline $\mathrm{N}$ & Number of turbines & \\
\hline $\mathrm{E}(\mathrm{H}, \mathrm{Q})$ & $\begin{array}{l}\text { Turbine efficiency curve, as a function of net head and flow (typically a function of turbine } \\
\text { type i.e. Francis, Pelton, etc.) }\end{array}$ & $\%$ \\
\hline$e_{\text {target }}$ & $\begin{array}{l}\text { Minimum efficiency threshold of turbines. Hydropower won't be generated if actual efficiency } \\
\text { is lower than } e_{\text {target }}\end{array}$ & $\%$ \\
\hline $\mathrm{Q}_{w}$ & Floodgates max hydraulic capacity & $\mathrm{m}^{3} / \mathrm{s}$ \\
\hline \multicolumn{3}{|c|}{ Reservoir topological data } \\
\hline$K_{T}$ & Identifier of the project downstream of turbine flow discharge & \\
\hline$K_{E}$ & Identifier of the project downstream of e-flow discharge & \\
\hline$K_{S}$ & Identifier of the project downstream of spills discharge & \\
\hline $\mathrm{R}$ & Position of the reservoir in the simulation sequence $\left(1^{\text {st }}, 2^{\text {nd }}, \ldots\right)$ & \\
\hline
\end{tabular}


Table SI-2. Sequence of calculations performed by the reservoir simulator model at a given time-step

\begin{tabular}{|c|c|c|c|}
\hline Value & Description & Calculation & Units \\
\hline $\mathrm{Q}_{\mathrm{u}}(t)$ & $\begin{array}{l}\text { Sum of upstream outflows (turbine, e- } \\
\text { flows and/or spills) directed to the } \\
\text { reservoir }\end{array}$ & & {$\left[\mathrm{m}^{3} / \mathrm{s}\right]$} \\
\hline$S_{t}$ & Reservoir storage at the beginning of step $t$ & & {$\left[\mathrm{~m}^{3}\right]$} \\
\hline$A_{\mathrm{t}}$ & $\begin{array}{l}\text { Area of reservoir at the beginning of step } \mathrm{t} \text {, } \\
\text { calculated from area volume curve }\end{array}$ & $A\left(\mathrm{~S}_{\mathrm{t}}\right)$ & {$\left[\mathrm{m}^{2}\right]$} \\
\hline $\mathrm{L}$ & Reservoir losses as evaporation & $\mathrm{A}_{\mathrm{t}} * \mathrm{ET}$ & {$\left[\mathrm{m}^{3}\right]$} \\
\hline $\mathrm{Va}_{t}$ & $\begin{array}{l}\text { Total available water for allocation } \\
\text { without restrictions at the reservoir, during } \\
\text { step t }\end{array}$ & $\begin{array}{l}\max \left(0, \mathrm{~S}_{\mathrm{t}}+\left(\mathrm{Q}_{\mathrm{a}}(\mathrm{t})+\mathrm{Q}_{\mathrm{u}}(\mathrm{t})\right) * \Delta \mathrm{t}-\mathrm{L}(\mathrm{t})-\right. \\
\left.\mathrm{V}_{\text {buffer }}\right)\end{array}$ & {$\left[\mathrm{m}^{3}\right]$} \\
\hline $\mathrm{Vr}_{t}$ & $\begin{array}{l}\text { Total available water for allocation, from } \\
\text { the buffer zone, during step t }\end{array}$ & $\begin{array}{l}\left(\mathrm{V}_{\text {buffer }}-V_{\text {min }}\right) * \mathrm{~B}_{\mathrm{c}}: \text { if } \mathrm{Va}_{t}>0 \\
\left(\mathrm{~S}_{\mathrm{t}}+\mathrm{Q}_{\mathrm{a}}(\mathrm{t}) * \Delta \mathrm{t}-\mathrm{L}(\mathrm{t})-V_{\min }\right) * \mathrm{~B}_{\mathrm{c}}: \text { if } \mathrm{Va}_{t}=0\end{array}$ & \\
\hline $\mathrm{Vu}_{t}$ & $\begin{array}{l}\text { Total available water for allocation during } \\
\text { step t }\end{array}$ & $\mathrm{Vr}_{t}+\mathrm{Va}_{t}$ & \\
\hline $\mathrm{Z}_{\mathrm{t}}$ & $\begin{array}{l}\text { Reservoir water level at the beginning of } \\
\text { step t, calculated from volume elevation } \\
\text { curve }\end{array}$ & $Z\left(\mathrm{~S}_{\mathrm{t}}\right)$ & masl \\
\hline$\Delta \mathrm{Z}_{\mathrm{t}}$ & Working water head at step t. & $\mathrm{Z}_{\mathrm{t}}-\mathrm{Z}_{0}$ & {$[\mathrm{~m}]$} \\
\hline $\mathrm{H}_{\mathrm{f}}$ & Friction losses & $\Delta \mathrm{Z}_{\mathrm{t}} * \mathrm{f}$ & {$[\mathrm{m}]$} \\
\hline RPE & $\begin{array}{l}\text { Expected generation at step t, expressed as } \\
\text { a percentage of installed capacity. }\end{array}$ & $\begin{array}{l}\text { User defined function of local (i.e. reservoir storage } \\
\text { at previous timestep, instream flow requirement } \\
\text { downstream of turbines, etc) or system-level state } \\
\text { variables or context variables (i.e. system level } \\
\text { demand, ENSO signal, etc.). See paper Figure } 3\end{array}$ & {$[\%]$} \\
\hline $\mathrm{V}_{\mathrm{pt}}$ & $\begin{array}{l}\text { Release requirement, to fulfill } 100 \% \text { of } \\
\text { expected energy generation at step t, } \\
\text { operating at target efficiency }\end{array}$ & $\begin{array}{c}\mathrm{RPE} * \mathrm{P} * 1 \mathrm{e} 6 /( \\
\left(9801 *\left(\Delta \mathrm{Z}_{\mathrm{t}}-\mathrm{H}_{\mathrm{f}}\right) * \mathrm{e}_{\text {target }}\right) \\
* \Delta \mathrm{t}\end{array}$ & {$\left[\mathrm{m}^{3}\right]$} \\
\hline $\mathrm{Vd}_{\mathrm{t}}$ & $\begin{array}{l}\text { Total water available during step t, after } \\
\text { the allocation of demands with higher } \\
\text { priorities than hydropower }\end{array}$ & $\max \left(0, V u_{t}-D_{t}-V_{\text {eco }, 1}\right)$ & {$\left[\mathrm{m}^{3}\right]$} \\
\hline$V_{\text {te }}$ & Effective turbined volume during step t & $\min \left(V_{p t}, V d_{t} t\right)$ & {$\left[\mathrm{m}^{3}\right]$} \\
\hline$Q_{\text {te }}$ & Effective turbined flow during step $\mathrm{t}$ & $\mathrm{V}_{\mathrm{te}} / \Delta \mathrm{t}$ & {$\left[\mathrm{m}^{3} / \mathrm{s}\right]$} \\
\hline $\mathrm{Vs}_{\mathrm{t}}$ & $\begin{array}{l}\text { Water available after hydropower is } \\
\text { allocated. }\end{array}$ & $V \mathrm{~d}_{\mathrm{t}}-V t_{e}$ & {$\left[\mathrm{~m}^{3}\right]$} \\
\hline$V_{\mathrm{w}}$ & $\begin{array}{l}\text { Controlled spill volume (floodgates } \\
\text { operation) }\end{array}$ & $\begin{array}{l}\qquad V_{w}=\min \left(R_{w}^{1 / B_{w}}, \mathrm{Q}_{w} * \Delta t\right) \\
\text { with: } \\
R_{w}=\max \left(0, \mathrm{~V} s_{\mathrm{t}}-\mathrm{V}_{w 0}\right): \text { Volume above the } \\
\text { floodgates operational level }\end{array}$ & {$\left[\mathrm{m}^{3}\right]$} \\
\hline $\mathrm{Vs}_{\mathrm{w}}$ & $\begin{array}{l}\text { Water available after controlled spill is } \\
\text { allocated. }\end{array}$ & $\mathrm{Vs}_{\mathrm{t}}-\mathrm{V}_{\mathrm{w}}$ & {$\left[\mathrm{m}^{3}\right]$} \\
\hline $\mathrm{V}_{\text {spill }_{\mathrm{t}}}$ & Total spill during step $\mathrm{t}$ & $\max \left(0, V s_{w}-V c\right)$ & {$\left[\mathrm{m}^{3}\right]$} \\
\hline
\end{tabular}




\begin{tabular}{|c|l|c|c|}
\hline Value & Description & Calculation & Units \\
\hline $\mathrm{P}_{\mathrm{n}}$ & $\begin{array}{l}\text { Net power output if available flow is } \\
\text { turbined given } H_{n, t}, \text { using } \mathrm{n} \text { turbines. }\end{array}$ & $\gamma * \frac{\mathrm{Q}_{\mathrm{te}}}{\mathrm{n}} * \mathrm{H}_{\mathrm{n}, \mathrm{t}} * \mathrm{e}_{\mathrm{t}, \mathrm{n}}$ \\
\hline $\mathrm{e}_{\mathrm{t}, \mathrm{n}}$ & $\begin{array}{l}\text { Actual Turbine/Generator efficiency given } \\
\left(\Delta \mathrm{Z}_{\mathrm{t}}-\mathrm{H}_{\mathrm{f}}\right) \text { and flow }=\frac{Q_{t e}}{n} \text {. See example in }\end{array}$ & $\begin{array}{l}\text { Figure SI-2. } \\
\text { If efficiency is lower than a target } \\
\text { efficiency, is adopted as } 0 .\end{array}$ & \multicolumn{1}{c|}{$\mathrm{e}_{\mathrm{n}}$} \\
\hline $\mathrm{P}_{\mathrm{t}}$ & $\begin{array}{l}\text { Average power output during step } \mathrm{t} \\
\text { if } \mathrm{e}_{\mathrm{n}}<\mathrm{e}_{\mathrm{target}}\end{array}$ & $\%$ \\
\hline $\mathrm{S}_{\mathrm{t}+1}$ & $\begin{array}{l}\text { Reservoir storage at the beginning of step } \\
\mathrm{t}+1\end{array}$ & $\max \left(\mathrm{P}_{1}, 2 * \mathrm{P}_{2}, \ldots \mathrm{N} * \mathrm{P}_{\mathrm{N}}\right)$ & {$[\mathrm{Mw}]$} \\
\hline
\end{tabular}

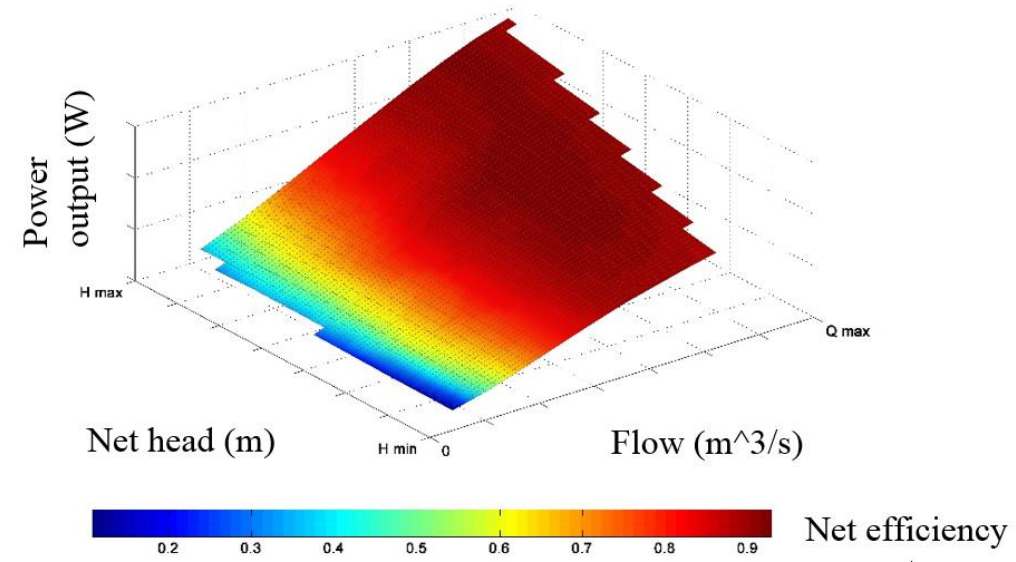

Figure SI-2. Example of a turbine power-efficiency function (Francis type) 